\title{
THz-Wave Generation from GaP THz Photonic Crystal Waveguides under Difference-Frequency Mixing
}

\author{
Kyosuke Saito $^{1}$, Tadao Tanabe ${ }^{2}$, Yutaka Oyama ${ }^{1}$ \\ ${ }^{1}$ Department of Materials Science, Graduate School of Engineering, Tohoku University, Sendai, Japan \\ ${ }^{2}$ Institute of Multidisciplinary Research for Advanced Materials, Tohoku University, Sendai, Japan \\ Email: k-saito@material.tohoku.ac.jp
}

Received June 21, 2012; revised July 27, 2012; accepted August 11, 2012

\begin{abstract}
$\mathrm{GaP}$ terahertz (THz) two-dimensional (2D) photonic crystal (PC) waveguides with line defects were fabricated using inductively-coupled plasma reactive-ion etching (ICP-RIE) in $\mathrm{Ar} / \mathrm{Cl}_{2}$ gas chemistries. THz-wave generation from the fabricated PC waveguides was demonstrated under collinear phase-matched difference-frequency generation (DFG), using Cr:Forsterite (Cr:F) lasers as the incident source. We compared the THz-wave output characteristics of the PC waveguides with that of GaP planar waveguides. The collinear phase-matching conditions in the DFG process were satisfied for 300- and 500- $\mu \mathrm{m}$-wide PC waveguide structures at 0.7 and $0.6 \mathrm{THz}$, respectively. The additional output peaks that appeared near the edge of the photonic band gap, around $0.5 \mathrm{THz}$, were attributed to the guiding modes in the PC waveguide; no such peaks appeared in the non-patterned ridge waveguides. These experimental results suggest that the phonon-polariton confinement in THz-PC waveguides based on the GaP crystal could be used to enhance the nonlinear optical effect for THz-wave generation.
\end{abstract}

Keywords: THz-Wave Generation; Photonic Crystal Waveguide; Nonlinear Optical Effect

\section{Introduction}

Terahertz-wave (THz-wave) sources have attracted a great deal of attention because the $\mathrm{THz}$ frequency region has a number of important potential applications in the medical and imaging fields [1,2]. Many coherent $\mathrm{THz}$ sources, based on photoconductive antenna, quantum cascade lasers, and non-linear optical effects, such as difference-frequency generation (DFG), optical parametric oscillation, and optical rectification, have been developed for these applications [3-17]. In particular, DFG can be used to produce tunable, narrow linewidth, high-power THz-waves, with ns-pulsed and continuous wave (CW) operation at room temperature $[18,19]$. This DFG process is suitable for wave sources used in $\mathrm{THz}$ telecommunications and in-situ security screening.

A GaP crystal is an attractive nonlinear optical material for coherent THz-wave generation via the DFG process, due to its transparent properties in the infrared and $\mathrm{THz}$ regions and relatively high conversion efficiency due to $\mathrm{THz}$ phonon-polariton excitation [7-10,12-14]. Our previous works have demonstrated THz-wave generation from bulk GaP crystals under small-angle, noncollinear $\chi(2)$ phase-matched DFG conditions [7-9]. Using a THz-wave source, a frequency-tunable $\mathrm{THz}$ spectrometer was constructed to study the $\mathrm{THz}$ spectra of biomolecules (e.g. sugars, nucleosides, nucleotides, and amino acids) [20-22]. When the cross-section size of the $\mathrm{GaP}$ rod-waveguide structure coincides with the wavelength of the $\mathrm{THz}$ wave, the $\mathrm{THz}$ wave is confined in the waveguide, thus, inducing a change in the refractive index. As a result, $\mathrm{THz}$ waves are produced under a collinear $\chi(2)$ phase-matching configuration. We have reported THz-wave generation from $\mathrm{GaP}-\mathrm{THz}$ rod and rib-waveguide structures and confirmed the enhancement of the conversion efficiency in the DFG process, using the wave-guiding effect for $\mathrm{THz}$ waves [23,24].

Recently, photonic crystal (PC) waveguides have been investigated because they allow the optical properties of the material to be modified. PC components have been developed in the optical region [25-28]. PCs in the THz region are also of interest because they could contribute to the development of $\mathrm{THz}$ technology. In contrast to passive devices, such as filters and PC fibers, few active devices have been investigated [29-33]. PC-embedded waveguides in the $\mathrm{THz}$ region make it possible to enhance the nonlinear optical effect, such as DFG in GaP, due to the strong phonon-polariton confinement in the PC waveguides.

In this paper, we fabricated two-dimensional (2D) photonic structures in $\mathrm{GaP}$ waveguides using inductivelycoupled plasma reactive-ion etching (ICP-RIE). The THz-wave output characteristics of the PC waveguides 
were compared with those of GaP planar waveguides.

\section{Experiment}

GaP-PC waveguides were fabricated using the ICP-RIE technique on an undoped semi-insulating (001) GaP wafer (Sumitomo Metal Mining, Co. Ltd.; resistivity: $>10^{5}$ W cm; thickness: $300 \mathrm{~mm}$ ). The fabrication process involved spin-coating an XP KMPR-1025 negative photoresist onto a $60-\mu \mathrm{m}$-thick $\mathrm{GaP}$ wafer, patterned using $\mathrm{UV}$ (i-line) exposure. Using a $\mathrm{Cl}_{2} / \mathrm{Ar}$ plasma chemistry in the ICP-IRE process, etch rates as high as $2 \mathrm{~mm} \cdot \mathrm{min}^{-1}$ were achieved to fabricate the PC waveguide structures. The waveguide samples were cleaved into $10 \times 10 \mathrm{~mm}$ rectangles, along the $<110>$ crystal direction. No antireflection coating was applied to the input or output surfaces of the waveguide. Figure 1 shows a scanning electron microscopy (SEM) image of one of the fabricated PC waveguides, with line defects in the G-K direction. Holes were arranged in a triangular lattice, with a lattice constant, a, of $200 \mathrm{~mm}$; the radius of the air holes, r, was 82 $\mathrm{mm}$. By removing rows of holes, we created 300- and 500-mm-wide line-defect waveguides along the $\mathrm{G}$ and $\mathrm{K}$ directions, respectively.

We calculated the photonic band diagram of the PC slab without line defects using the plane-wave expansion method (Figure 2(a)). The lowest stop band-gap region was estimated to exist in the frequency range of 0.4 to $0.55 \mathrm{THz}$ for transverse electric (TE) polarization. We also calculated the $\mathrm{THz}$ transmission spectra for the $\mathrm{THz}-$ PC slab and waveguide using three-dimensional (3-D) finite-difference time-domain (3D-FDTD) simulations, shown in Figure 2(b). The modeled structures were the same as the PC without line defects and the 300-mmwide PC waveguide. The THz-wave transmission spectra indicated high transmittance in the stop band-gap region for the PC waveguide. Thus, this suggests the existence of defect modes originating from the line defects in this frequency region.

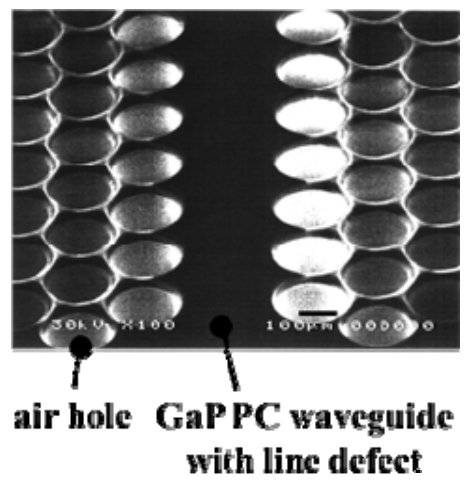

Figure 1. SEM bird's-eye-view photograph of fabricated GaP photonic crystal (PC) waveguide with line defect widths of $300 \mu \mathrm{m}$.

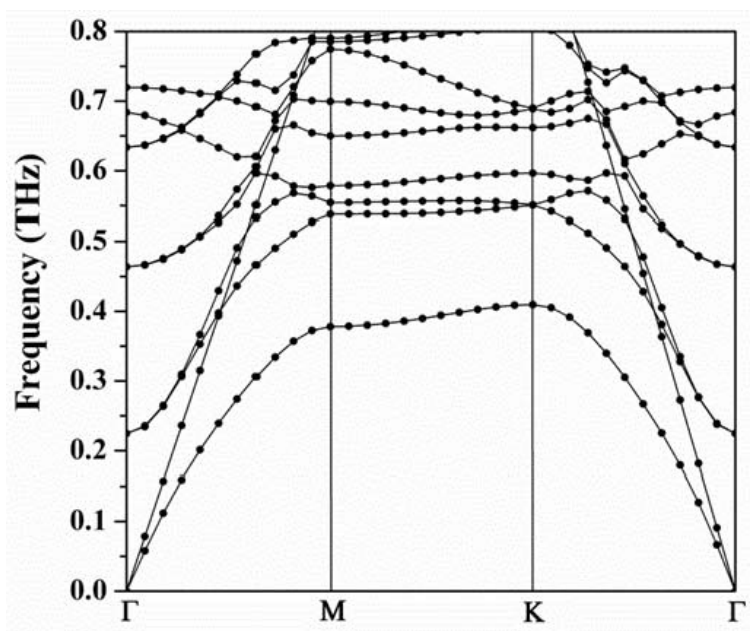

(a)

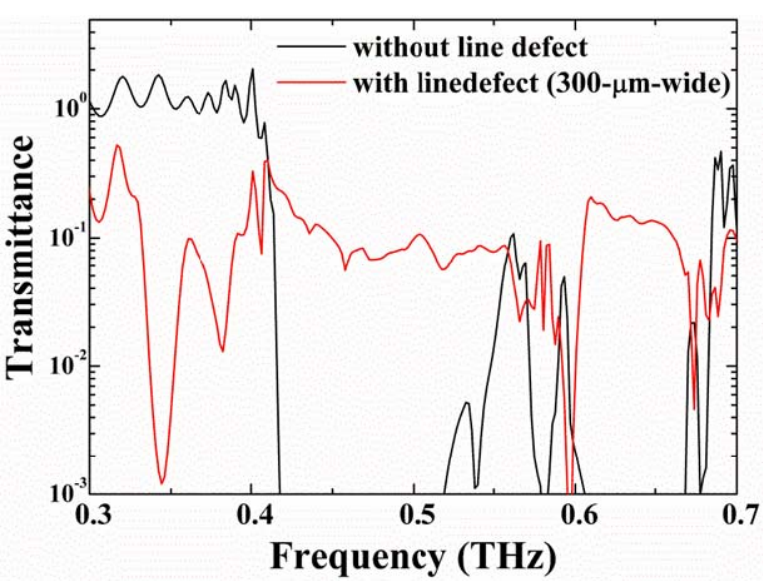

(b)

Figure 2. Calculated results of (a) photonic band diagram without line defects and (b) THz transmission spectra for the THz PC slab and waveguide ( 300 - $\mu \mathrm{m}$-wide linewidth).

Figure 3 illustrates a schematic of the experimental setup used for DFG of THz waves in GaP-PC waveguides, where $\mathrm{Cr}$ :Forsterite $(\mathrm{Cr}: \mathrm{F})$ lasers were used as the incident source. Both the pump and signal beams were delivered from two-channel Cr:F lasers (LOTIS TII) pumped with a double-pulse Q-switched Nd:YAG laser (repetition rate: $10 \mathrm{~Hz}$, wavelength: $1064 \mathrm{~nm}$ ). The wavelength of the pump source was fixed at $1216 \mathrm{~nm}$; the signal source was variable from 1217 to $1221 \mathrm{~nm}$, which corresponded to a frequency difference of 0.2 to $1.0 \mathrm{THz}$. The pulse energies of the pump and signal sources were adjusted to $0.5 \mathrm{~mJ}$ (pulse width: $22 \mathrm{~ns}$; beam diameter: 1 $\mathrm{mm}$ ). The pump and signal beam were collinearly combined using a beamsplitter and then directed to the input edge of the waveguide; the propagation direction of the two beams was parallel to the G-K direction. The generated $\mathrm{THz}$ wave was collected by a high-density polyethylene (HDPE) lens and detected by a liquid-helium-cooled Si-bolometer. 


\section{The Results and Discussions}

THz-wave generation was carried out under collinear phase-matched DFG in GaP-PC waveguides. Figures 4(a) and (b) display the output characteristics of the generated $\mathrm{THz}$ wave from the 500- and 300- $\mu \mathrm{m}-\mathrm{GaP}-\mathrm{PC}$ waveguides, respectively. Figure 4(c) shows the THz-wave output from the $\mathrm{GaP}$ ridge waveguide, which had a thickness and width of $300 \mathrm{~mm}$. The polarization of the generated $\mathrm{THz}$ wave is TE polarization. In the instances where the frequency of the signal source was varied at $1 \mathrm{GHz}$ steps with respect to the fixed-frequency of the pump source, a $100 \mathrm{GHz}$ bandwidth was observed at the peak frequency positions for phase matching, for both the $0.6 \mathrm{THz}$ (corresponding to the $500-\mathrm{mm}$-wide waveguide) and $0.7 \mathrm{THz}$ (300- $\mu \mathrm{m}$-wide waveguide) outputs. The peak frequency shift is due to the difference in the effective index for $\mathrm{THz}$ waves in the PC waveguides.

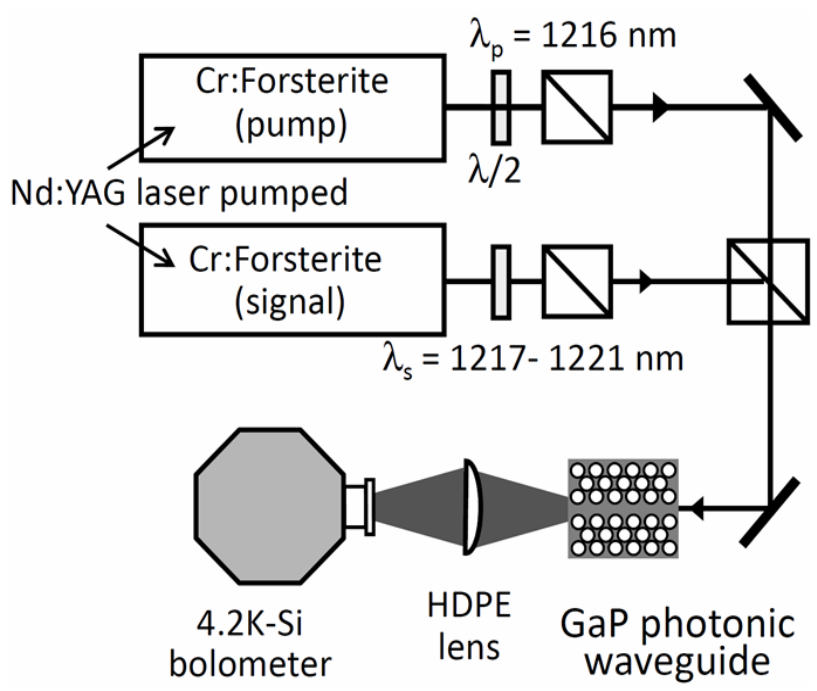

Figure 3. Schematic diagram of the experimental setup for THz-wave generation in GaP PC waveguides.

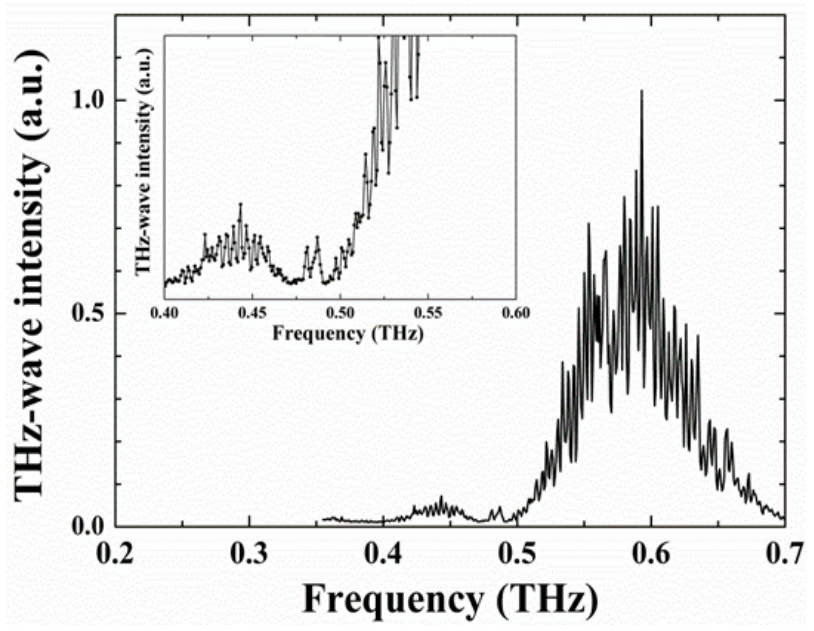

(a)

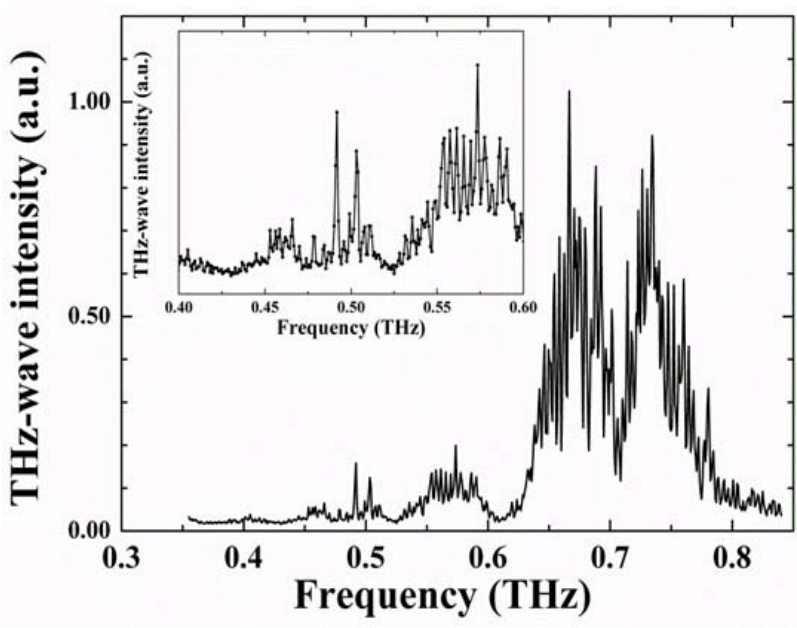

(b)

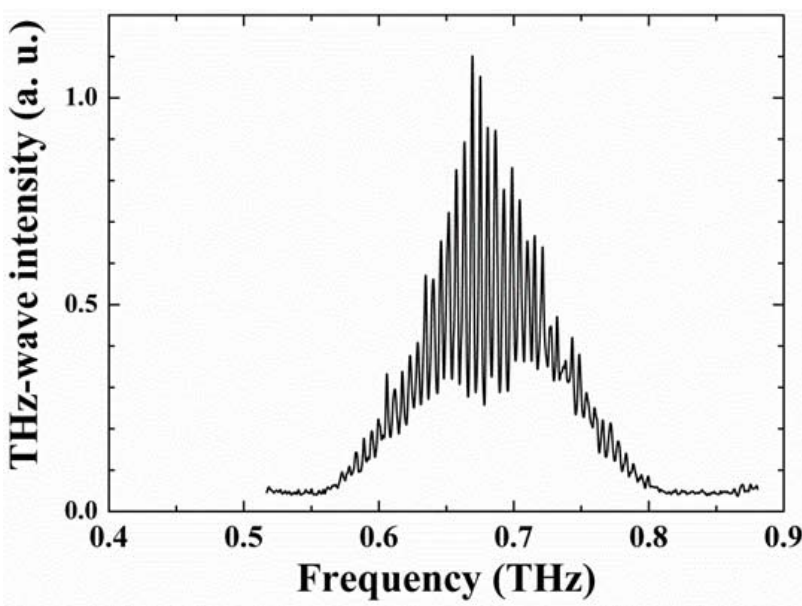

(c)

Figure 4. Frequency dependence of THz-wave output intensity from GaP-PC waveguides and non-patterned ridge waveguides with (a) line defect width of $500 \mu \mathrm{m}$; (b) line defect width of $300 \mu \mathrm{m}$; and (c) ridge width of $300 \mu \mathrm{m}$. The insets in Figures 4(a) and (b) show enlarged view of the figures around $0.5 \mathrm{THz}$.

These bandwidths arise from the phase mismatch in the DFG process. The linewidth of THz wave is determined by that of incident infrared sources $(500 \mathrm{MHz})$. Therefore, THz wave linewidth obtained by difference frequency process was about $1 \mathrm{GHz}$. The generated $\mathrm{THz}$ wave was tunable within the spectral bandwidth. The oscillation of the $\mathrm{THz}$ output power for both waveguides was observed due to the interference of the $\mathrm{THz}$ waves between the input and output faces of the waveguide. Therefore, we confirmed that the GaP-PC line-defect waveguides act as $\mathrm{THz}$ waveguides. For the $300-\mathrm{mm}$ wide $\mathrm{PC}$ waveguide, a decrease in the $\mathrm{THz}$ output power appeared around $0.7 \mathrm{THz}$; this can be attributed to the transmission characteristics of the PC waveguide. The conversion efficiencies of generated $\mathrm{THz}$ waves from the 
waveguides were in the order of $10^{-4} \%$. This value is comparable as other nonlinear optical techniques such as optical parametric oscillation, optical rectification, and photoconductive antenna.

As mentioned above, the stop-band frequency was expected to be in the frequency range of 0.40 to $0.55 \mathrm{THz}$. For PC structures with line defects, the guiding modes should be shown in the stop-band frequency range. The insets of Figures 4(a) and (b) show the enlarged view around $0.5 \mathrm{THz}$. For the two $\mathrm{PC}$ waveguides, a $\mathrm{THz}$ output peak with narrow spectral bandwidth appeared around $0.5 \mathrm{THz}$, although phase matching was not satisfied in this frequency region. The intensity of the peaks for the $500-\mathrm{mm}-\mathrm{PC}$ waveguide was larger than that for the $300-\mathrm{mm}-\mathrm{PC}$ waveguide. Because no additional peak was observed in the phase-mismatched region for the non-patterned ridge waveguide (Figure 4(c)), the $\mathrm{THz}$ output characteristics were attributed to defect modes in the stop-band frequency region. The defect modes have a low group velocity, which corresponds to a large effective index of refraction, compared with non-patterned $\mathrm{THz}$ waveguides. This suggests that the modal dispersions of the defect modes contributed to the phasematching condition around $0.5 \mathrm{THz}$.

\section{Conclusion}

We have fabricated THz-GaP waveguides based on 2D PCs produced by reactive-ion etching in $\mathrm{Ar} / \mathrm{Cl}_{2}$ plasma chemistries. $\mathrm{THz}$ waves were generated from the fabricated PC waveguides with line defects. The collinear phase-matching conditions in the DFG process were satisfied for 300- and 500-mm-wide PC waveguide structures at 0.7 and $0.6 \mathrm{THz}$, respectively. Furthermore, the $\mathrm{THz}$ output peak with narrow spectral width appeared around $0.5 \mathrm{THz}$ for both PC waveguides. This frequency region is within the stop-band (non phase-matching) region, as suggested by the numerical calculations. These $\mathrm{THz}$ output peaks were unique for these THz-PC waveguide structures. Due to the phonon-polariton confinement in GaP-PC line-defect waveguides, our experimental results indicated the possibility of enhancing the nonlinear optical effect for THz-wave generation using THzPC waveguides based on the GaP crystal.

\section{Acknowledgements}

The authors would like to thank to T. Sasaki, K. Suto, and J. Nishizawa of the Semiconductor Research Institute for the discussion on the experimental results. We also would like to thank to T. Kimura of the Semiconductor Research Institute for help with waveguide fabrication. The part of this research was supported by KDDI foundation.

\section{REFERENCES}

[1] J. Nishizawa, "Open-Up a New Field in Tera-Hertz Band," Journal of Acoustical Society of Japan, Vol. 57, No. 2, 2001, pp. 163-169.

[2] M. Tonouchi, "Cutting-Edge Terahertz Technology," Nature Photonics, Vol. 1, No. 2, 2007, pp. 97-105. doi: $10.1038 /$ nphoton. 2007.3

[3] D. A. Auston, K. P. Cheung and P. R. Smith, "Picosecond Photoconducting Hertzian Dipoles," Applied Physics Letters, Vol. 45, No. 3, 1984, pp. 284-286. doi:10.1063/1.95174

[4] P. U. Jepsen, R. H. Jacobsen and S. R. Keiding, "Generation and Detection of Terahertz Pulses from Biased Semiconductor Antennas," Journal of the Optical Society of America B, Vol. 13, No. 11, 1996, pp. 2424-2436. doi:10.1364/JOSAB.13.002424

[5] M. Tani, S. Matsuura, K. Sakai and S. Nakashima, "Emission Characteristics of Photoconductive Antennas Based on Low-Temperature-Grown GaAs and Semi-Insulating GaAs," Applied Optics, Vol. 36, No. 30, 1997, pp. 7853-7859. doi:10.1364/AO.36.007853

[6] J. Faist, F. Capasso, D. L. Sivco, C. Sirtori, A. L. Hutchinson and A. Y. Cho, "Quantum Cascade Laser," Science, Vol. 264, No. 5158, 1994, pp. 553-556. doi: $10.1126 /$ science. 264.5158 .553

[7] T. Tanabe, K. Suto, J. Nishizawa, T. Kimura and K. Saito, "Frequency-Tunable High-Power Terahertz Wave Generation from GaP," Journal of Applied Physics, Vol. 93, No. 8, 2003, pp. 4610-4615. doi:10.1063/1.1560573

[8] T. Tanabe, K. Suto, J. Nishizawa, K. Saito and T. Kimura, "Tunable Terahertz Wave Generation in the 3- to 7-THz region from GaP," Applied Physics Letters, Vol. 83, No. 2, 2003, pp. 237-239. doi:10.1063/1.1592889

[9] T. Tanabe, K. Suto, J. Nishizawa, K. Saito and T. Kimura, "Frequency-Tunable Terahertz Wave Generation via Excitation of Phonon-Polaritons in GaP," Journal of Physics D: Applied Physics, Vol. 36, No. 8, 2003, pp. 953-957. doi:10.1088/0022-3727/36/8/302

[10] F. De Martini, "Infrared Generation by Coherent Excitation of Polaritons," Physical Review B, Vol. 4, No. 12, 1971, pp. 4556-4578. doi:10.1103/PhysRevB.4.4556

[11] K. Kawase, M. Mizuno, S. Sohoma, H. Takahashi, T. Taniuchi, Y. Urata, S. Wada, H. Tashiro and H. Ito, "Difference-Frequency Terahertz-Wave Generation from 4-Dimethylamino-N-methyl-4-stilbazolium-tosylate by Use of an Electronically Tuned Ti: Sapphire Laser," Optics Letters, Vol. 24, No. 15, 1999, pp. 1065-1067. doi:10.1364/OL.24.001065

[12] T. Taniuchi and H. Nakanishi, "Collinear Phase-Matched Terahertz-Wave Generation in GaP Crystal Using a DualWavelength Optical Parametric Oscillator," Journal of Applied Physics, Vol. 95, No. 12, 2004, pp. 7588-7591. doi:10.1063/1.1751238

[13] W. Shi and Y. J. Ding, "Tunable Terahertz Waves Generated by Mixing Two Copropagating Infrared Beams in GaP," Optics Letters, Vol. 30, No. 9, 2005, pp. 1030-1032. doi:10.1364/OL.30.001030 
[14] I. Tomita, H. Suzuki, H. Ito, H. Takenouchi, K. Ajito, R. Rungsawang and Y. Ueno, "Terahertz-Wave Generation from Quasi-Phase-Matched GaP for $1.55 \mu \mathrm{m}$ Pumping," Applied Physics Letters, Vol. 88, No. 7, 2006, Article ID: 071118. doi:10.1063/1.2174832

[15] K. Kawase, J. Shikata and H. Ito, "Terahertz Wave Parametric Source," Journal of Physics D: Applied Physics, Vol. 35, No. 3, 2002, pp. R1-R14. doi:10.1088/0022-3727/35/3/201

[16] X.-C. Zhang, Y. Jin and X. F. Ma, "Coherent Measurement of $\mathrm{THz}$ Optical Rectification from Electro-Optic Crystals," Applied Physics Letters, Vol. 61, No. 23, 1992, pp. 2764-2766. doi:10.1063/1.108083

[17] A. Nahata, A. S. Weling and T. F. Heinz, "A Wideband Coherent Terahertz Spectroscopy System Using Optical Rectification and Electro-Optic Sampling," Applied Physics Letters, Vol. 69, No. 16, 1996, pp. 2321-2323. doi:10.1063/1.117511

[18] J. Nishizawa, T. Tanabe, K. Suto, Y. Watanabe, T. Sasaki and Y. Oyama, "Continuous-Wave Frequency-Tunable Terahertz-Wave Generation from GaP," IEEE Photonics Technology Letters, Vol. 18, No. 19, 2006, pp. 2008-2010. doi:10.1109/LPT.2006.882278

[19] T. Tanabe, J. Nishizawa, K. Suto, Y. Watanabe, T. Sasaki and Y. Oyama, "Terahertz Wave Generation from GaP with Continuous Wave and Pulse Pumping in the $1-1.2$ $\mu \mathrm{m}$ Region," Materials Transactions, Vol. 48, No. 5, 2007, pp. 980-983. doi:10.2320/matertrans.48.980

[20] J. Nishizawa, T. Sasaki, K. Suto, T. Tanabe, K. Saito, T. Yamada and T. Kimura, "THz Transmittance Measurements of Nucleobases and Related Molecules in the 0.4to 5.8-THz Region Using a GaP THz Wave Generator," Optics Communication, Vol. 246, No. 1-3, 2005, pp. 229239. doi:10.1016/j.optcom.2004.10.076

[21] J. Nishizawa, K. Suto, T. Sasaki, T. Tanabe and T. Kimura, "Spectral Measurement of Terahertz Vibrations of Biomolecules Using a GaP Terahertz-Wave Generator with Automatic Scanning Control," Journal of Physics D: Applied Physics, Vol. 36, No. 23, 2003, pp. 2958-2961. doi:10.1088/0022-3727/36/23/015

[22] J. Nishizawa, T. Sasaki, K. Suto, T. Tanabe, T. Yoshida, T. Kimura and K. Saito, "Frequency-Tunable TerahertzWave Generation from GaP Using Cr:Forsterite Lases," International Journal of Infrared and Millimeter Waves, Vol. 27, No. 6, 2006, pp. 779-789. doi: $10.1007 / \mathrm{s} 10762-006-9125-4$

[23] J. Nishizawa, K. Suto, T. Tanabe, K. Saito, T. Kimura and Y. Oyama, "THz Generation from GaP Rod-Type WaveGuides," IEEE Photonics Technology Letters, Vol. 19,
No. 3, 2007, pp.143-145 doi:10.1109/LPT.2006.890098

[24] K. Saito, T. Tanabe, Y. Oyama, K. Suto and J. Nishizawa, "Terahertz-Wave Generation by GaP Rib Waveguides via Collinear Phase-Matched Difference-Frequency Mixing of Near-Infrared Lasers," Journal of Applied Physics, Vol. 105, No. 6, 2009, Article ID: 063102. doi:10.1063/1.3094012

[25] Y. Jiang, W. Jiang, L. Gu, X. Chen and R. T. Chen, "80Micron Interaction Length Silicon Photonic Crystal WaveGuide Modulator," Applied Physics Letters, Vol. 87, No. 22, 2005, Article ID: 221105. doi:10.1063/1.2138367

[26] S. Guo and S. Albin, "Numerical Techniques for Excitation and Analysis of Defect Modes in Photonic Crystals," Optics Express, Vol. 11, No. 9, 2003, pp. 1080-1089. doi:10.1364/OE.11.001080

[27] A. J. Danner, J. J. Raftery, P. O. Leisher and K. D. Choquette, "Single Mode Photonic Crystal Vertical Cavity Lasers," Applied Physics Letters, Vol. 88, No. 9, 2006, Article ID: 091114. doi:10.1063/1.2181268

[28] T. A. Birks, D. Mogilevtsev, J. C. Knight and P. St. J. Russell, "Dispersion Compensation Using Single-Material Fibers," IEEE Photonics Technology Letters, Vol. 11, No. 6, 1999, pp. 674-676. doi:10.1109/68.766781

[29] T. D. Drysdale, I. S. Gregory, C. Baker, E. H. Linfield, W. R. Tribe and D. R. S. Cumming, "Transmittance of a Tunable Filter at Terahertz Frequencies," Applied Physics Letters, Vol. 85, No. 22, 2004, pp. 5173-5175. doi:10.1063/1.1829798

[30] K. Takagi, K. Seno and A. Kawasaki, "Fabrication of a Three-Dimensional Terahertz Photonic Crystal Using Monosized Spherical Particles," Applied Physics Letters, Vol. 85 , No. 17,2004 , pp. 3681-3683. doi:10.1063/1.1812375

[31] F. Miyamaru, M. Tanaka and M. Hangyo, "Effect of Hole Diameter on Terahertz Surface-Wave Excitation in MetalHole Arrays," Physical Review B, Vol. 74, No. 15, 2006, Article ID: 153416. doi:10.1103/PhysRevB.74.153416

[32] A. L. Bingham and D. Grischkowsky, "High Q, One-Dimensional Terahertz Photonic Waveguides," Applied Physics Letters, Vol. 90, No. 9, 2007, Article ID: 091105. doi:10.1063/1.2710002

[33] G. Diwa, A. Quema, E. Estacio, R. Probre, H. Murakami, S. Ono and N. Sarukura, "Photonic-Crystal-Fiber Pigtail Device Integrated with Lens-Duct Optics for Terahertz Radiation Coupling," Applied Physics Letters, Vol. 87, No. 15, 2005, Article ID: 151114. doi:10.1063/1.2093941 